\title{
GOBERNANZA Y RECTORÍA DE LA CALIDAD EN LOS SERVICIOS DE SALUD EN EL PERÚ
}

\author{
Óscar Ugarte-Ubilluz ${ }^{1, a}$
}

\begin{abstract}
RESUMEN
El cuidado de las personas y de su salud es una función primaria de la familia y de la sociedad como lo demuestran estudios sobre los humanos primitivos, así como en el Perú prehispánico. La conquista y los siglos posteriores de colonización quebraron la forma tradicional del cuidado de las personas, reemplazando la solidaridad social por acciones de caridad principalmente de órdenes religiosas que propiciaron hospicios luego denominados hospitales. Durante la colonia y hasta principios del siglo XX el cuidado de los enfermos siguió siendo responsabilidad de las instituciones de caridad, como las Beneficencias creadas luego de la independencia. Los derechos sociales como la educación y la salud recién surgen en las primeras décadas del pasado siglo, plasmándose en la Constitución de 1933. Sin embargo, tanto en esa Constitución como en la de 1979 y la de 1993 el derecho a la educación fue reconocido más plenamente, siendo más limitado en salud. La ley de Aseguramiento Universal en Salud del 2009 propone garantizar para todos el derecho al acceso a servicios de salud con calidad, como parte del derecho a la salud en sentido amplio. Las limitaciones actuales obligan a redefinir el derecho de todas las personas al cuidado integral de su salud y la rectoría del Estado para garantizarlo.
\end{abstract}

Palabras clave: política de salud; Prestación de Atención de Salud; Perú (fuente: DeCS BIREME).

\section{GOVERNANCE AND STEWARDSHIP OF QUALITY IN HEALTH SERVICES IN PERU}

\begin{abstract}
The care of people and their health is a primary function of the family and of society as shown by studies on primitive humans, as well as in pre-Hispanic Peru. The conquest and subsequent centuries of colonization fractured the traditional way of caring for people, replacing social solidarity with charity actions mainly from religious orders that provided hospices later called hospitals. During the colony and until the beginning of the 20th century, the care of the sick continued to be the responsibility of charitable institutions, such as the Charities created after independence. Social rights such as education and health only emerged in the first decades of the last century and were enshrined in the 1933 Constitution. However, both in that Constitution as in those from 1979 and 1993, the right to education was recognized more fully, while the right to heath was limited. The Universal Health Coverage Act of 2009 propounds guaranteeing the right to access quality healthcare services for everybody, as part of the right to health in the broadest sense. The current limitations force us to redefine the right of every citizen to comprehensive care of their health and the State's guidance to guarantee it.
\end{abstract}

Keywords: Health policy; Healthcare delivery; Perú (source: MeSH NLM).

\section{ANTECEDENTES HISTÓRICOS DEL CUIDADO DE LA SALUD}

El cuidado de la salud de las personas históricamente ha sido responsabilidad de las familias y de la sociedad, tanto en épocas primitivas como en civilizaciones más desarrolladas. Yuval Noah Harari (2016), en su libro «Sapiens - Una breve historia de la humanidad», describe características de los neardentales 70 mil años antes de nuestra era ${ }^{(1)}$.
«Estos humanos eran más musculosos que los sapiens, poseían un cerebro mayor y estaban mejor adaptados a los climas fríos. Empleaban utensilios y fuego, eran buenos cazadores y aparentemente cuidaban de sus enfermos $y$ débiles. (Los arqueólogos han descubierto huesos de neandertales que vivieron durante muchos años con impedimentos físicos graves, que son prueba de que eran cuidados por sus parientes)».

\footnotetext{
Instituto de Gobierno, Universidad de San Martín de Porres. Lima, Perú.

a MBA de Salud, Universidad de Ciencias Aplicadas. Lima, Perú.

Recibido: 24/04/2019 Aprobado: 06/06/2019 En línea: 28/06/2019
}

Citar como: Ugarte-Ubilluz Ó. Gobernanza y rectoría de la calidad en los servicios de salud en el Perú. Rev Peru Med Exp Salud Publica. 2019;36(2):296-303. doi: http://dx.doi.org/10.17843/rpmesp.2019.362.4495. 


\section{EL CUIDADODELASALUDENELPERÚPREHISPÁNICO}

Igualmente, en el Perú prehispánico el cuidado de las personas fue integral. Guamán Poma de Ayala describe la organización social en el Tawantinsuyo por grupos etáreos y funciones ${ }^{(2)}$, que incluían el cuidado de la salud y de los enfermos, en los siguientes términos:

- Auca Camayoc: jóvenes de 25 a 33 años, preparados para la guerra, servían obligatoriamente hasta los 50 años en la labranza, oficios diversos, minería, apoyo a autoridades.

- Puric Macho: desde los 51 hasta los 78 años, ya no iban a la guerra pero estaban obligados a servir en todos los mandados.

- Rocto Macho: desde los 80 hasta los 100 años, muy temidos y obedecidos, encargados de dar consejos y doctrinas, así como castigar a quien incumpla.

- Uncoc Runa: personas enfermas o discapacitadas de cualquier edad, les daban casa y sementeras, y personas a su servicio.

- Sayapayac: jóvenes de 18 a 20 años, servían como mensajeros y para hacer encargos de un pueblo a otro, cuidaban el ganado, llevaban la comida y servían a las autoridades del pueblo.

- Mactakuna: jóvenes de 12 a 18 años, encargados de guardar el ganado y cazar aves con hondas y redes, para utilizar su carne y sus plumas para los Incas y autoridades. Les enseñaban humildad y obediencia.

- Tocllacoc wanrakuna: niños cazadores, de nueve a 12 años, cazaban pajaritos menudos como jilgueros, picaflor o palomas para hacer charqui con su carne y utilizar las plumas para confección de tejidos finos.

- Pucllacoc wamrakuna: niños juguetones, de cinco a nueve años, servían a sus padres y a sus madres en su casa, encargados de jugar con los chicos más pequeños o niños huérfanos.

- Llullu Wamrakuna: niños de teta que comienzan a gatear y hasta los cinco años, que deben jugar con otros niños y deben ser cuidados para que no se caigan ni se quemen y puedan crecer bien.

- Kirawpi wawakuna, niños de teta, recién nacidos que están en la cuna, hasta la edad de un año, es obligatorio que lo atienda la madre que tiene que darle la leche y no otra persona, salvo que sea huérfano.

En el Tawantinsuyo había claras orientaciones para garantizar un adecuado cuidado de la salud, a través de ordenanzas aprobadas por el Inca, como por ejemplo con relación a la alimentación ${ }^{(2)}$ :
«Mandamos en todo el reino haya abundancia de comida $y$ que se siembre muy mucho maíz y papas y ocas $y$ que hayan cavi,caya, chuño, tamos chochoca y quinua, olluco, mashua; todas las comidas hasta las yerbas, yuyos acuáticos lo sequen, para que tengan qué comer todo el año y se siembre de comunidad y sapci de maíz, papas, aji, magno. ; y algodón y cojan flor de pavaua queveucha y otras hojas para teñir colores.»

"Mandamos en este reino en los pueblos han de comeren la plaza pública caciques principales, indios chicos y grandes para que se alleguen todos los pobres y huérfanos, viudas, enfermos, viejos, ciegos y tullidos, peregrinos caminantes.»

"De cómo tenían costumbre de purgarse cada mes con purga que ellos llaman bilca tawri (purgante de tawri), Con ello tenían mucha fuerza para pelear y aumentaban salud y comían con mucho gusto.»

Igualmente, había ordenanzas para las personas dedicadas especialmente al cuidado de la salud (2):

«Mandamos que los barberos y seruxanos (médicos y cirujanos), hanpi camayoc, circa, quichicauan (sangrador) y curan con yerbas. A estos dichos les llamaban hampi camayoc y a las dichas parteras beatas comadres llamaban huahua huachachic (partera), huicza allichac hanpi camayoc (la curandera que arregla estómago).»

«Mandamos que las dichas amas de güérfanos, waccha rurocha nunochic, sean reservados de todo y le den otra niña grande para que le ayude a criar. Estos se llamaban mamacona (señoras).»

Según Poma de Ayala, como se observa en la Figura 1, en el Tawantinsuyo los enfermos eran atendido por su familia o la comunidad.

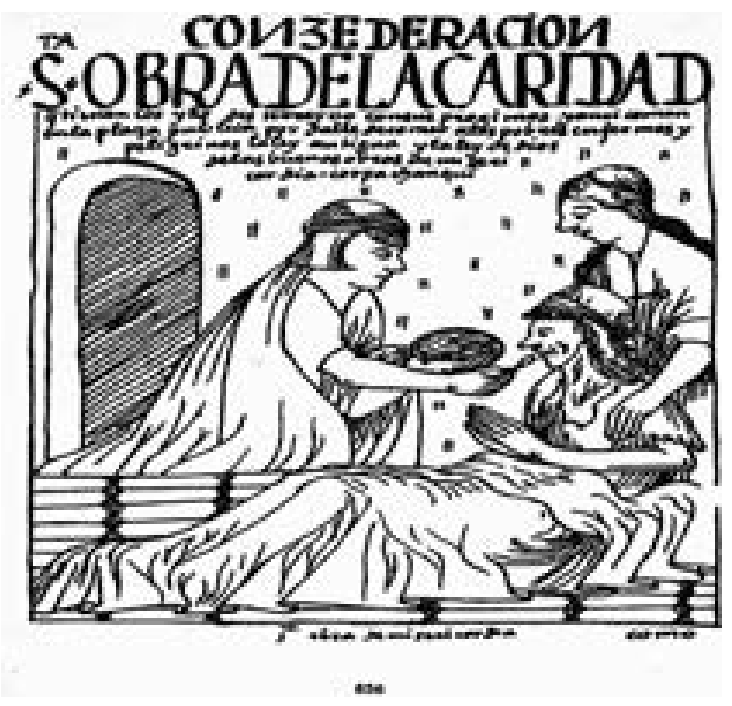

Figura 1. Ilustración de Guamán Poma de Ayala de la atención de un enfermo en el Tawantinsuyo 
Como se puede apreciar, la organización social del Tawantinsuyo incorporaba el cuidado integral de las personas o, como se diría actualmente, un régimen de seguridad social integral.

\section{LA CONQUISTA QUEBRÓ EL SISTEMADE CUIDADO DE LAS PERSONAS EXISTENTE EN EL TAWANTINSUYO}

La conquista quebró el ordenamiento social existente con consecuencias muy graves en la cohesión social, la producción, la educación, la salud y otras condiciones sociales.

Durante la Colonia el estado Virreynal no asumió responsabilidades sociales con las personas, tanto la educación como la salud pasaron a ser responsabilidad exclusivamente de las familias. Los únicos servicios de salud los organizaron órdenes religiosas por caridad para los pobres. Así se creó el hospital de San Andrés, primer hospital de Sudamérica, fundado en 1548 por congregaciones religiosas para atender a los españoles pobres; y el de Santa Ana, para atender principalmente a los indígenas prohibidos de atenderse en el SanAndrés. Las familias pudientes recibían atención en sus respectivas casas por parte de los escasos médicos existentes formados en Europa. Los hospitales fundados por caridad eran financiados por familias ricas, con donaciones en efectivo y posteriormente propiedades.

Como se observa en la Figura 2, que muestra un mapa de la ciudad de Lima, que data de 1746, dentro de la ciudad amurallada ya había ocho hospitales: Espíritu Santo, San Juan de Dios, San Pedro, San Andrés, Santa Ana, San Bartolomé, de los Incurables (luego Santo Toribio de Mogrovejo) y el de Convalecientes ${ }^{(3)}$.
En 1821, existían en el Perú cincuenta hospitales, once de los cuales se ubicaban en Lima, y los demás en el resto del país.

Ya durante la República se crearon las Beneficencias, para tener un sistema institucionalizado que administre las donaciones de las familias adineradas. Se crearon en Lima (1834), Cusco (1835), Trujillo (1847), Huancayo (1847), Arequipa (1848), Callao (1849), y demás ciudades importantes del país. El Estado no tenía servicios de salud propios, salvo las iniciales sanidades militares desarrolladas como necesidad en las confrontaciones bélicas. El modelo de cuidados de la salud de la colonia, basado en la caridad, continuó durante la República hasta el siglo $\mathrm{XX}$, cuando recién empiezan a reconocerse progresivamente derechos sociales que se plasman en la Constitución de 1933.

\section{EL CUIDADO DE LA SALUD EN EL SIGLO XX}

\section{LA CONSTITUCIÓN DE 1933: RECONOCIMIENTO DE DERECHOS SOCIALES Y CREACIÓN DEL MINISTERIO $D E S A L U D$}

Los derechos sociales como el derecho al trabajo, a la educación y a la salud surgieron en el mundo en el siglo XIX con posterioridad a los derechos civiles y políticos. En el Perú tales derechos fueron reconocidos recién en las primeras décadas del siglo XX, como por ejemplo la jornada de ocho horas en 1918. La Constitución de 1933 es la primera que reconoce los derechos sociales, en especial educación y más tímidamente en salud ${ }^{(4)}$ :

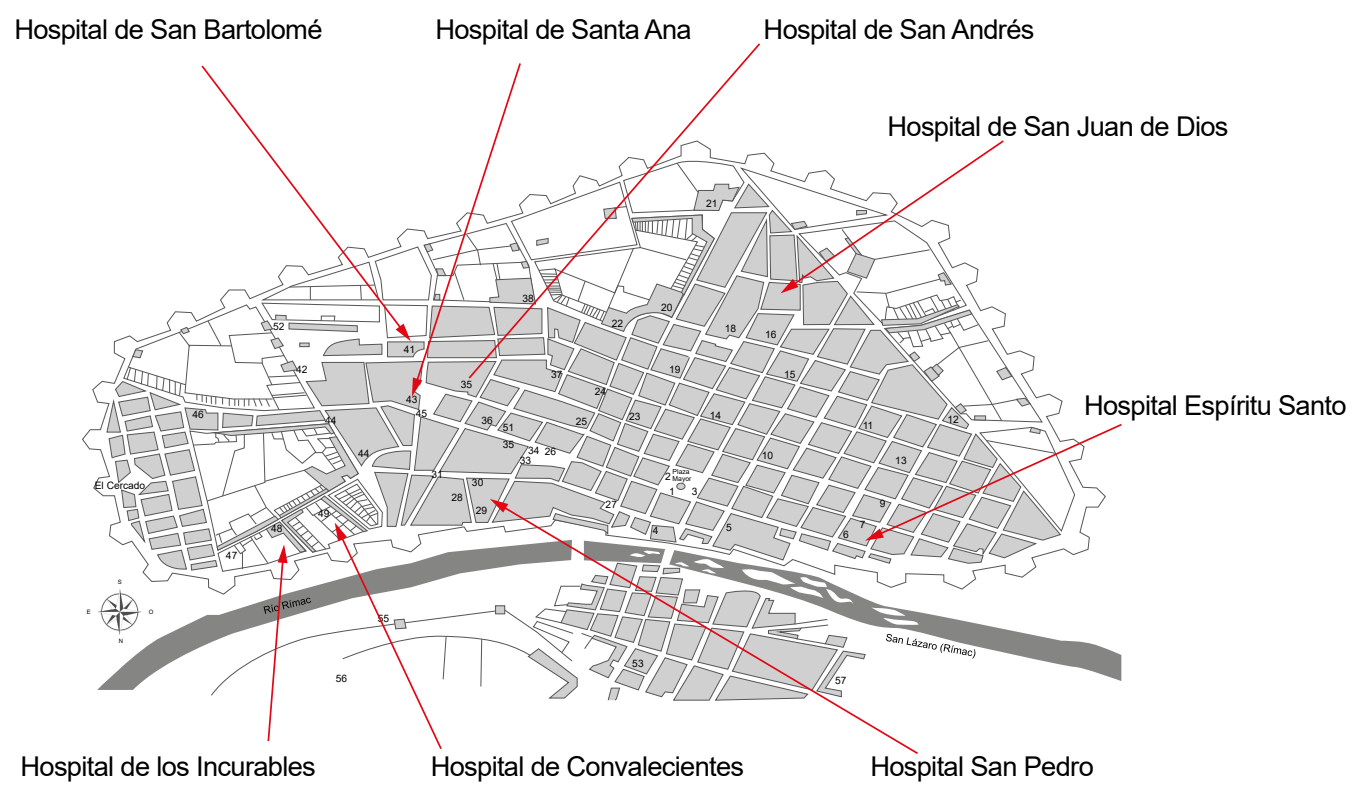

Fuente: Charles Walker (2012), «Colonialismo en Ruinas: Lima frente a terremoto y tsunami de 1746».

Figura 2. Mapa del cercado de la ciudad de Lima en 1746 
- La enseñanza primaria es obligatoria y gratuita (artículo 72).

- El Estado fomenta la enseñanza en sus grados secundario y superior, con tendencia a la gratuidad (artículo 75).

- El Estado defiende el derecho del niño a la vida del hogar, a la educación, a la orientación vocacional, y a la amplia asistencia cuando se halle en situación de abandono, de enfermedad o de desgracia (artículo 52).

Como se observa, se reconoce el derecho amplio en educación, pero en salud se limita a la infancia abandonada.

En 1934 se creó el Ministerio de Educación y en 1935 el Ministerio de Salud Pública, Trabajo y Previsión Social, durante el gobierno del general Oscar R. Benavides. La primera estructuración del Ministerio de Salud fue a través de la creación de Servicios Nacionales, orientados a combatir las principales epidemias como la malaria, la tuberculosis, la lepra, las enfermedades venéreas y otras. Experiencias pioneras fueron del Dr. Hugo Pesce en la provincia de Andahuaylas ${ }^{(5)}$ y el Dr. Manuel Núñez Butrón en Puno ${ }^{(6)}$.

CREACIÓN DE LA SEGURIDAD SOCIAL EN 1936: RECONOCIMIENTO DEL DERECHO A LA SALUD A QUIENES TIENEN TRABAJO ESTABLE

En 1936 se promulgó la ley 8433 creando el Seguro Social Obrero (SSO), primera institución de previsión social con el aporte de patronos y trabajadores de la naciente industria peruana. Con ese fondo se construyó en 1941 el Hospital Obrero de Lima, hoy denominado Hospital Almenara, así como una red de establecimientos menores para la atención de estos asegurados.

Así surgió la tercera tendencia en la organización de servicios de salud, en adición a los hospitales de caridad administrados por las Beneficencias y a los servicios del naciente Ministerio de Salud. Esta fue la adaptación al Perú de las orientaciones impulsadas por Bismark en Alemania en el siglo XIX.

Con esa misma orientación en 1948 se creó el Seguro Social del Empleado (SSE), que motivó en los años 50 la construcción del Hospital del Empleado en Lima, luego denominado Hospital Rebagliatti, y otros en el interior del país. En 1975 se fusionaron ambos seguros.

\section{CREACIÓN DE LAS SANIDADES DE SALUD DE LAS FUERZAS ARMADAS Y DE LA POLICIIA NACIONAL}

Si bien los servicios de salud castrenses crecieron con las Fuerzas Armadas desde el inicio de la república, eran limitados a servicios de cirugía de guerra requeridos en el período de la Independencia y posteriormente en el largo período de guerra entre caudillos militares hasta mediados del siglo XIX.
Recién a mediados del siglo $X X$ se crean verdaderos sistemas de atención para los militares y sus familiares. Expresión de ello fue, en la década del 50, la construcción del Hospital del Ejército, el Hospital Naval, el Hospital de la Policía Nacional y posteriormente el Hospital de la Fuerza Aérea. Años después se construyeron otros servicios castrenses en el resto del país, de menor capacidad resolutiva.

Este subsistema de servicios de salud castrenses es en realidad una variante del derecho relacionado con el trabajo, porque se sustenta en las contribuciones que aportan los integrantes de dichas instituciones; pero con organización autónoma.

\section{CREACIÓN DE SERVICIOS DE SALUD POR INICIATIVA PRIVADA}

Paralelamente a la creación de los subsistemas de salud descritos anteriormente, desde la segunda mitad del siglo XIX surgieron servicios de salud privados. La primera Clínica privada en el Perú fue la Maison de Santé, fundada en 1866 por la Sociedad Francesa de Beneficencia para ciudadanos franceses residentes en el Perú.

Con la misma orientación, a principios de 1900 fue creada la clínica Italiana, por la Sociedad Italiana de Beneficencia para ciudadanos italianos residentes en el país. Igualmente, en 1921 se creó la Clínica Angloamericana, por iniciativa de ciudadanos británicos y norteamericanos que llegaron al Perú con las primeras empresas mineras, petroleras y comerciales. Inicialmente dicha clínica funcionó en Bellavista, pero luego del terremoto de 1940 se construyó el local de Miraflores. En los 40 se crean la Clínica Delgado y la Clínica Losada, como iniciativa privada familiar; y también la Clínica Good Hope, por iniciativa de la Iglesia Adventista.

En 1956 se creó la Clínica Stella Maris, regentada por religiosas católicas. En 1958 se crea la Clínica San Felipe y en 1959 se crea la Clínica Internacional, por el Grupo Brescia. En la década del 60 se crean la Clínica Javier Prado, el Policlínico Peruano-Japonés. Y en los años 70 se crearon la Clínica Ricardo Palma, la Clínica San Borja y la Clínica Tezza.

Asimismo, en los 80 se fundaron la Clínica Vesalio, la Clínica Montefiori y la Clínica Santa Teresa. En los 90 se creó la Clínica Limatambo, Oncosalud, la Clínica El Golf, la Clínica San Pablo y la Clínica San Judas Tadeo.

En los 2000 se crea la Clínica Centenario, de la Asociación Peruano-Japonesa, la Clínica San Gabriel, la Clínica Jesús del Norte, la Clínica Jockey Salud y otras. 
En menor proporción, se crearon clínicas privadas en la mayoría de departamentos del país, principalmente por iniciativa de grupos de médicos independientes. De igual manera, las grandes empresas mineras, petroleras, azucareras y de otras ramas de la producción crearon servicios privados para sus respectivos trabajadores.

\section{LA CONSTITUCIÓN DE 1979 AMPLÍA LOS DERECHOS EN EDUCACIÓN Y SALUD}

Esta Constitución amplía el derecho a la educación y a la salud:

- La educación inicial, primaria y secundaria son obligatorias. En las instituciones del Estado, la educación es gratuita (artículo 17).

- En las universidades públicas el Estado garantiza el derecho a educarse gratuitamente a los alumnos que mantengan un rendimiento satisfactorio y no cuenten con los recursos económicos necesarios (artículo 17).

- El Estado garantiza el derecho de todos a la seguridad social. La ley regula el acceso progresivo a ella y su financiación (artículo 12).

- La seguridad social tiene como objeto cubrir los riesgos de enfermedad, maternidad, invalidez, desempleo, accidente, vejez, muerte, viudez, orfandad y cualquier otra contingencia susceptible de ser amparada conforme a ley (artículo 13).

- Todos tienen derecho a la protección de la salud integral y el deber de participar en la promoción y defensa de su salud, la de su medio familiar y de la comunidad (artículo 15).

- El Poder Ejecutivo señala la política nacional de salud. Controla y supervisa su aplicación. Fomenta las iniciativas destinadas a ampliar la cobertura y calidad de los servicios de salud dentro de un régimen pluralista. Es responsable de la organización de un sistema nacional descentralizado y desconcentrado, que planifica y coordina la atención integral de la salud a través de organismos públicos y privados, y que facilita a todos el acceso igualitario a sus servicios, en calidad adecuada y con tendencia a la gratuidad. La ley norma su organización y funciones (artículo 16).

Como se observa, la Constitución de 1979 amplió los derechos en educación y salud, superando a la del 33. Correspondía posteriormente formular las políticas que garanticen esos derechos, particularmente a acceder a servicios de salud de calidad y con tendencia la gratuidad.

LA CONSTITUCIÓN DE 1993 MANTUVO LOS DERECHOS EN EDUCACIÓN, PERO RESTRINGIÓ LOS DERECHOS EN SALUD

Esta Constitución mantuvo en educación los mismos derechos ya reconocidos por la Constitución de 1979, pero retrocedió en salud:
- La educación inicial, primaria y secundaria son obligatorias. En las instituciones del Estado, la educación es gratuita (artículo 17).

- En las universidades públicas el Estado garantiza el derecho a educarse gratuitamente a los alumnos que mantengan un rendimiento satisfactorio y no cuenten con los recursos económicos necesarios (artículo 17)

Pero en salud se eliminó lo referente a garantizar el acceso de todos a los servicios de salud con tendencia a la gratuidad:

- Todos tienen derecho a la protección de su salud, la del medio familiar y la de la comunidad, así como el deber de contribuir a su promoción y defensa. La persona incapacitada para velar por sí misma a causa de una deficiencia física o mental tiene derecho al respeto de su dignidad y a un régimen legal de protección, atención, readaptación y seguridad (artículo 7 ).

- El Estado determina la política nacional de salud. El poder ejecutivo norma y supervisa su aplicación. Es responsable de diseñarla en forma plural y descentralizadora para facilitar a todos el acceso equitativo a los servicios de salud (artículo 9).

- El Estado reconoce el derecho universal y progresivo de toda persona a la seguridad social, para su protección frente a la contingencia que precise la ley y para la elevación de su calidad de vida (artículo 10).

- El Estado garantiza el libre acceso a prestaciones de salud y a pensiones, a través de entidades públicas, privadas o mixtas. Supervisa asimismo su eficaz cumplimiento (artículo 11).

Como se puede observar, la Constitución de 1993, todavía vigente, mantiene el reconocimiento del derecho universal a acceder a la seguridad social, pero condicionado al trabajo estable; y elimina la posibilidad de que las personas que no tengan trabajo estable puedan acceder a servicios de salud de calidad, con tendencia a la gratuidad, es decir con financiamiento subsidiado por el Estado, como decía la Constitución de 1979.

LA LEY 29344 DE ASEGURAMIENTO UNIVERSAL EN SALUD (AUS) AMPLÍA EL DERECHO DE TODOS A ACCEDER A SERVICIOS DE SALUD

EI AUS se propone garantizar el derecho pleno y progresivo a la salud de todas las personas residentes en el país:

- Derecho pleno y progresivo de toda persona a la salud (artículo 1).

- Acceder a un conjunto de prestaciones de salud de carácter preventivo, promocional, recuperativo y de 
rehabilitación, en condiciones adecuadas de eficiencia, equidad, oportunidad, calidad y dignidad (artículo 3).

- Financiamiento subsidiado para aquella población más pobre y vulnerable, así como mecanismos para evitar la selección adversa (artículo 4).

La ley del AUS se fundamenta en que el derecho a la salud no es completo si no se garantiza el acceso a servicios de salud en forma universal, tal como es el derecho a la educación. $\mathrm{Y}$ corresponde al estado subsidiarla para la población pobre y vulnerable.

Con la ley de aseguramiento universal del 2009 se abrió un proceso que ha permitido que un $88,2 \%$ de la población tenga el derecho de acceder a servicios de salud. Como se observa en la Figura 3, el $88,2 \%$ de la población ya está afiliada a un seguro de salud, mayoritariamente en el Seguro Integral de Salud (SIS) (52\%), en segundo lugar, EsSalud (30\%) y en tercer lugar el aseguramiento privado y las sanidades (6\%).

El incremento del aseguramiento público ha ido de la mano de un incremento del financiamiento público en salud, aunque no lo suficiente. Como se observa en la Figura 4 el financiamiento público en salud creció casi diez veces en cifras absolutas entre el 2000 y el 2019.

De otro lado, el financiamiento del SIS, es decir del costo variable del aseguramiento público, creció en ese mismo período de 131 millones de soles en el 2002 a 1,750 millones en el 2015, o sea 14 veces. Sin embargo, se redujo en el 2016 a 1550 millones y en el 2017 a 1400 millones de soles; creció en el 2018 a 2200 millones de soles y volvió a reducirse en el 2019 a 1750 millones.

El aseguramiento público tiene actualmente dos limitaciones importantes: de un lado, no ha logrado aún incorporar al $100 \%$ de la población; y de otro lado el plan de beneficios (PEAS), pese a haberse ampliado no tiene la amplitud del plan de beneficios de EsSalud. Por ello, el gran reto

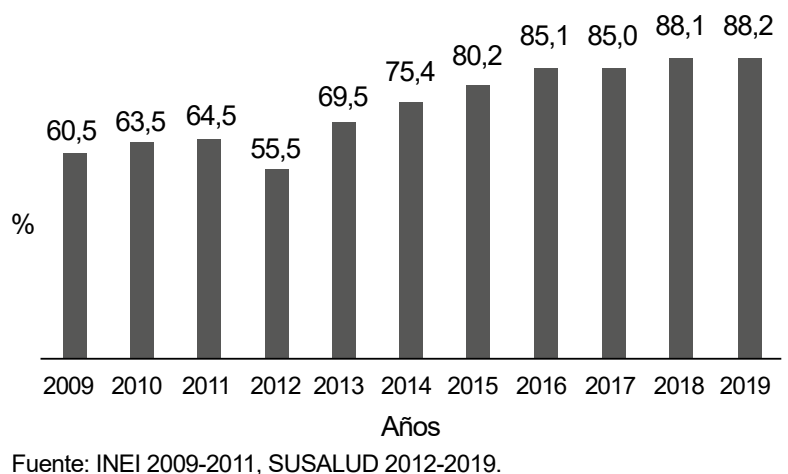

Fuente: INEI 2009-2011, SUSALUD 2012-2019.

Figura 3. Cobertura de Aseguramiento en Perú durante 2009-2019 del aseguramiento es incrementar el plan de beneficios y mejorar sustantivamente la oferta de servicios de salud.

\section{LA CALIDAD COMO ELEMENTO ESENCIAL DEL DERECHO A SERVICIOS DE SALUD CENTRADOS EN LA PERSONA}

La Organización Mundial de la Salud (OMS) ${ }^{(7)}$, señala que la calidad de la asistencia sanitaria es asegurar que cada persona reciba el conjunto de servicios diagnósticos y terapéuticos más adecuado para conseguir una atención óptima, teniendo en cuenta todos los factores y conocimientos del paciente y de la sociedad. Sus características esenciales son:

- $\quad$ Alto nivel de excelencia profesional.

- Uso eficiente de los recursos.

- Mínimo riesgo para el paciente.

- $\quad$ Alto grado de satisfacción del paciente.

- Valoración del impacto final en la salud.

Avedis Donabedian, uno de los principales estudiosos que han aportado a la calidad en salud, la define así: «La calidad de la atención consiste en la aplicación de la ciencia y la tecnología médicas en una forma que maximice sus beneficios para la salud sin aumentar en forma proporcional sus riesgos. El grado de calidad es por consiguiente la medida en que se espera que la atención suministrada logre el equilibrio más favorable de riesgos y beneficios».

Asimismo, define la gestión de la calidad como «el proceso de producción de un servicio de salud de acuerdo a los estándares previstos, garantizando la más alta eficiencia en el uso de los recursos, produciendo el menor riesgo posible para el usuario y procurando obtener el objetivo deseado y a la vez su máxima satisfacción» ${ }^{(8)}$.

Un aspecto fundamental de la calidad en servicios de salud es el respeto a los derechos de los usuarios. En Perú están contenidos en la ley 29414 del 2009 , que modificó y amplió el artículo 15 de la Ley General de Salud de 1996; referidos a cuatro grandes campos: acceso a los servicios de salud, acceso a la información, atención y recuperación de la salud, y consentimiento informado.

La ley del AUS creó la Superintendencia Nacional de Salud, cuya principal función es garantizar el cumplimiento de los derechos de los usuarios en salud. Luego fue fortalecida con el decreto legislativo (DL) 1158, que le otorgó esa función para todo tipo de servicios de salud y no sólo en el ámbito del aseguramiento. 


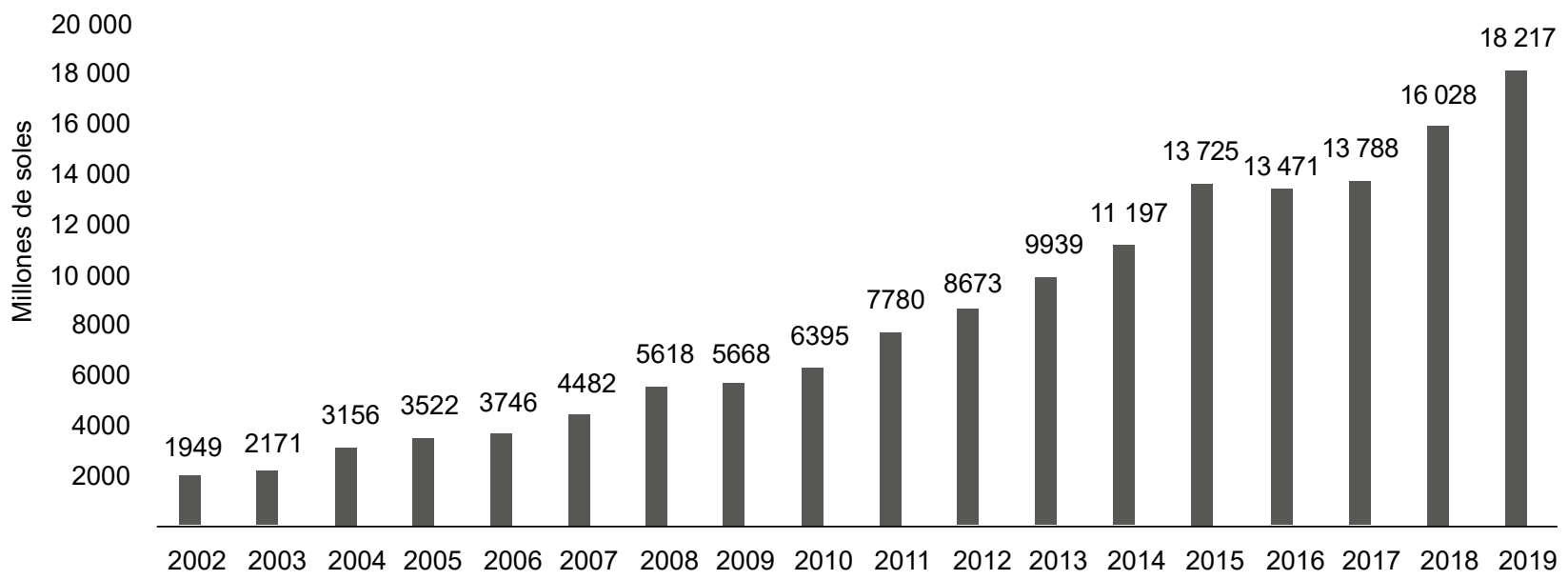

Fuente: Ministerio de Economía y Finanzas.

Figura 4. Evolución de los presupuestos en salud durante 2002-2019 en Perú.

\section{LA GOBERNANZA Y RECTORÍA EN EL DERECHO A SERVICIOS DE SALUD}

\section{AUTORIDAD SANITARIA Y RECTORÍA}

Según la OPS, la autoridad sanitaria es el custodio del bien público en salud y su objetivo primordial es la protección y la promoción de la salud de la población; y se expresa como la potestad del Estado para incidir sobre las funciones, responsabilidades y competencias sustantivas que le son propias e indelegables, a través del Ministerio o Secretaría de Salud de cada país. Por ello, define la rectoría en salud como el ejercicio de las responsabilidades y competencias sustantivas de la política pública en salud, en el contexto de las relaciones entre gobierno y sociedad en el Estado moderno ${ }^{(9)}$.

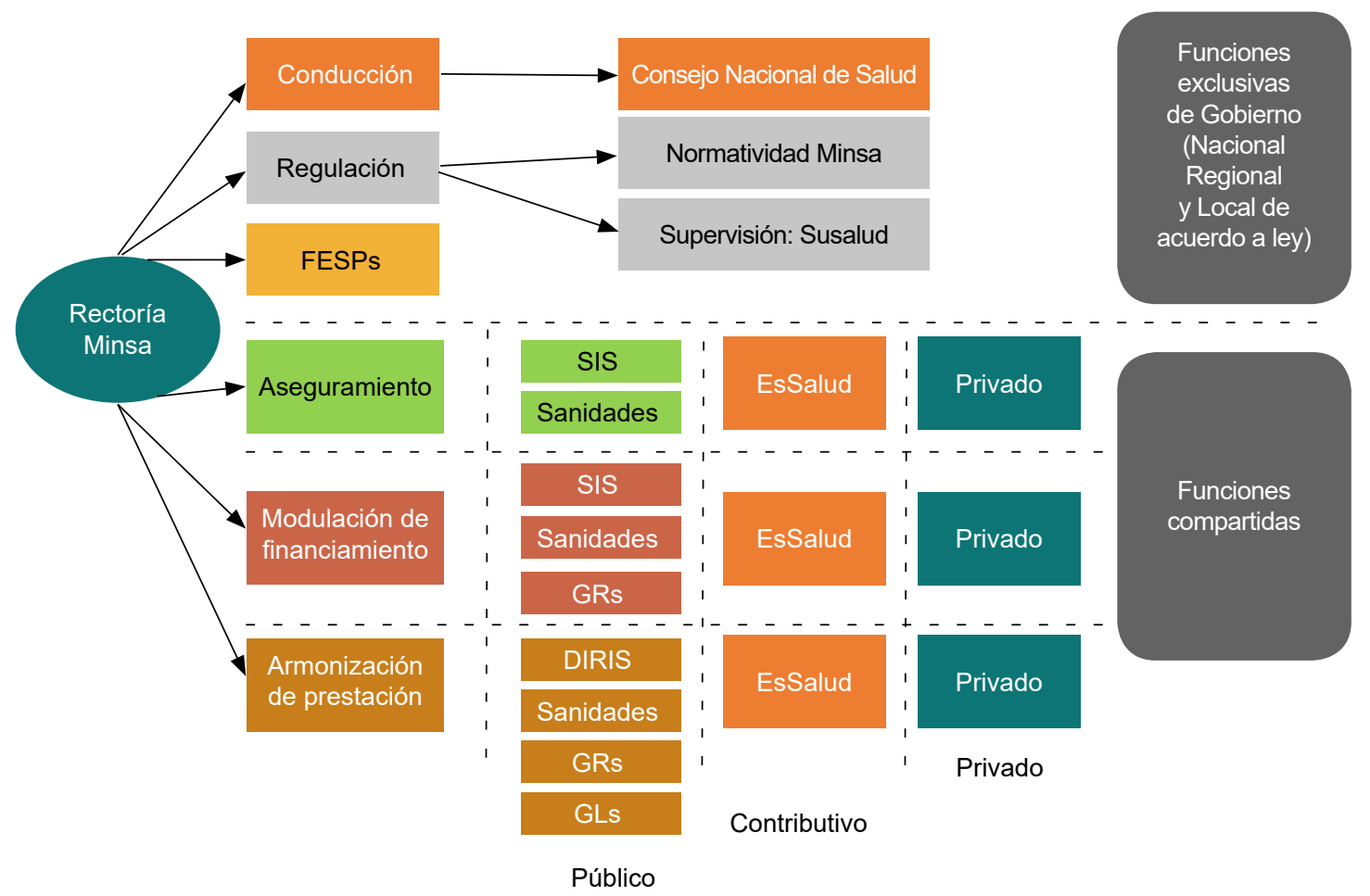

Fuente: Organización Panamericana de la Salud

SIS: Seguro Integral de Salud, FESPs: Funciones esenciales de salud pública, GRs: Gobiernos regionales, GLs: Gobiernos locales

Figura 5. Función Rectora de la Autoridad Sanitaria Nacional 


\section{DIMENSIONES DE LA RECTORÍA}

Con ese fin, la rectoría comprende seis grandes dimensiones o áreas de responsabilidad y de competencias institucionales (9): Conducción sectorial, regulación, modulación del financiamiento, garantía del aseguramiento, armonización de la provisión, y ejecución de las funciones de salud pública.

Como lo describe la OPS y se observa en la Figura 5, la provisión de servicios de salud es una función compartida por el MINSA, EsSalud, Sanidades y sector privado, sobre la cual el MINSA ejerce rectoría mediante la armonización de los servicios de prestación, a través de la normatividad y supervisión.

\section{RETOS PARA ALCANZAR EL PLENO DERECHO AL CUIDADO INTEGRAL DE LA SALUD COMO PARTE DE LOS OBJETIVOS DE DESARROLLO SOSTENIBLE AL 2030}

- Modificar nuestro marco legal incorporando el derecho de todos los ciudadanos al cuidado integral de la salud centrado en la persona.

- Lograr la cobertura sanitaria universal, incluida la protección contra los riesgos financieros, el acceso a servicios de salud esenciales de calidad y el acceso a medicamentos.
- Ampliar progresivamente elPEAS, comoloestablece el artículo 16 de la ley del AUS, de tal manera que el plan de beneficios del aseguramiento público sea similar a EsSalud, consolidando las ampliaciones ya logradas con los planes complementarios, el plan de salud escolar, la restitución del Plan Esperanza, cuidados en salud mental, etc.

- Ampliar el financiamiento per cápita del Seguro Integral de Salud (SIS), para hacerlo equivalente al financiamiento per cápita de EsSalud. Para ello, reglamentar y aplicar la Ley 29761 de financiamiento del aseguramiento público.

- Definir una ruta de integración/articulación de largo plazo de la Seguridad Social y del Aseguramiento Público.

- Definir e implementar un plan nacional de inversiones en infraestructura y equipamiento, priorizando el primer nivel y cerrando las brechas interregionales.

- Formular y desarrollar un plan nacional de recursos humanos, para garantizar una cobertura de profesionales de la salud por lo menos de 45 por 10 mil habitantes, superando el promedio actual de 31 priorizando las regiones más deficitarias.

- Impulsar la provisión adecuada de medicamentos y otros insumos estratégicos, fortaleciendo las compras corporativas del sector público y aprovechando las ventajas de ser un comprador monopsónico en determinados medicamentos.

- Fortalecer las capacidades gerenciales de las instituciones públicas, generando mayor eficiencia y un mejor desempeño en la atención a la ciudadanía.

- Fortalecimiento del rol rector del Ministerio de Salud en la conducción del proceso de Seguridad Social Universal.

\section{REFERENCIAS BIBLIOGRÁFICAS}

1. Harari YN. Sapiens, p. 27. Londres: Penguin Random House; 2016.

2. Guamán Poma de Ayala, Primer Nueva Corónica y Buen Gobierno. Siglo XXI/ IEP, Tomo I pp. 169-209. Lima 1980.

3. Walker C. Colonialismo en Ruinas: Lima frente a terremoto y tsunami de 1746. Lima: InstitutoFrancés de Estudios Andinos; 2012.

4. Perú, Congreso dela República[Internet]. Lima: Congreso de la República; 1933. Constitución de 1933. Disponible en: http://www.leyes.congreso.gob.pe/
Documentos/constituciones_ordenado/ CONSTIT_1933/Cons 1933 TEXTO.pdf

5. Barrio J. Anécdotas del Maestro Hugo Pesce. Lima: Instituto de Salud Hugo Pesce-INSAHP; 1988.

6. Neyra J. "Héroes de la salud pública en el Perú” Manuel Núñez Butrón. Rev Peru Med Exp Salud publica. 2005 22(2):148-9.

7. Organización Mundial de la Salud [Internet]. Ginebra: OMS; 2017. Boletin de la Organización Mundial de la Salud, Volumen 95, Número 5, mayo 2017.
Disponible en: https://www.who.int/ bulletin/volumes/95/5/es/

8. Donabedian A. La Calidad de la atención médica: definición y métodos de evaluación. México, DF: La Prensa Médica Mexicana; 1984

9. OPS. Función Rectora de la Autoridad Sanitaria Nacional, Ed. Especial $\mathrm{N}^{\circ} 17$. Washington DC, 2007.

\footnotetext{
Correspondencia: Oscar Ugarte

Correoelectrónico:ougarteu@gmail.com
} 